\title{
Association of antepartum and intrapartum SARS-CoV-2 infection on pregnancy outcomes in South African women, two observational studies
}

Marta Nunes $^{1}$, Stephanie Jones ${ }^{1}$, Renate Strehlau ${ }^{1}$, Vuyelwa Baba ${ }^{1}$, Zanele Ditse ${ }^{1}$, Kelly Da Silva ${ }^{1}$, Lane Bothma ${ }^{1}$, Natali Serafin ${ }^{1}$, Vicky Baillie ${ }^{1}$, Gaurav Kwatra ${ }^{1}$, Megan Burke ${ }^{1}$, Amy Wise ${ }^{1}$, Mary Adam ${ }^{1}$, Philiswa Mlandu ${ }^{2}$, Mpolokeng Melamu ${ }^{2}$, Juliette Phelp ${ }^{2}$, Wendy Fraser ${ }^{3}$, Colleen Wright ${ }^{3}$, Elizabeth Zell ${ }^{4}$, Yasmin Adam ${ }^{5}$, and Shabir Madhi ${ }^{1}$

${ }^{1}$ University of the Witwatersrand Faculty of Health Sciences

${ }^{2}$ Chris Hani Baragwaneth Academic Hospital, University of the Witwatersrand,

Johannesburg

${ }^{3}$ Lancet Laboratories

${ }^{4}$ Stat-Epi Associates, Inc.

${ }^{5}$ Chris Hani Baragwanath Academic Hospital and the University of the Witwatersrand

November 18, 2021

\begin{abstract}
Objective: Evaluate the impact of the timing of SARS-CoV-2 infection on pregnancy outcomes in a low-middle income setting. Design: two parallel, observational studies. Setting and population: pregnant women or women presenting for labour, enrolled between April-September 2020, in South Africa. Methods: i) longitudinal follow-up study of symptomatic or asymptomatic pregnant women investigated for SARS-CoV-2 infection antenatally, ii) cross-sectional study of SARS-CoV-2 infection at time of labour. SARS-CoV-2 infection was investigated by nucleic acid amplification test (NAAT). Main Outcome Measures: association of SARS-CoV-2 infection on nasal swab and birth outcomes. Results: Antenatally, 793 women were tested for SARS-CoV-2. Overall SARS-CoV-2 infection was confirmed in 138 women, including 119/275 with symptomatic illness (COVID-19) and 19/518 asymptomatic women; 493 women were asymptomatic and SARS-CoV-2 non-reactive. Women with COVID-19 were 1.66-times (95\%CI: 1.02, 1.71) more likely to have a low-birthweight newborn (30\%) compared to asymptomatic women without SARS-CoV-2 (21\%). Overall, 3117 women were tested for SARS-CoV-2 infection at delivery, including 1560 healthy women with an uncomplicated term delivery. Adverse birth outcomes or pregnancy-related complications were not associated with infection at delivery. Among women with SARS-CoV-2 infection at delivery, NAAT was reactive on 6/98 of maternal blood samples, 8/93 of cord-blood, 14/54 of placentas and 3/22 of nasopharyngeal swabs from newborns collected within 72-hours of birth. Conclusions: Antenatal, but not intrapartum, SARS-CoV-2 infection was associated with low-birthweight delivery. Maternal infection at the time of labour was associated with in utero foetal and placental infection, and possible vertical and/or horizontal viral transfer to the newborn.
\end{abstract}

\section{Hosted file}

MNH_text_bjog_15Nov2021.docx available at https://authorea.com/users/446790/articles/545951association-of-antepartum-and-intrapartum-sars-cov-2-infection-on-pregnancy-outcomes-insouth-african-women-two-observational-studies

\section{Hosted file}


MNH_Tables_BJ_Nov2021.docx available at https://authorea.com/users/446790/articles/545951association-of-antepartum-and-intrapartum-sars-cov-2-infection-on-pregnancy-outcomes-insouth-african-women-two-observational-studies 